\title{
Novel Analysis and Visualization of Chemical Events for Public Health Surveillance
}

\author{
Michael J. Henry*, Lauren Charles-Smith, Kyungsik Han and Courtney D. Corley \\ Visual Analytics, Pacific Northwest National Laboratory, Richland, WA, USA
}

\section{Objective}

Pacific Northwest National Laboratory hosted an intern-based web application development contest in the summer of 2016 centered around developing novel chemical surveillance applications to aid in health situational awareness. Making up the three teams were three graduate students $(n=9)$ from various US schools majoring in nonpublic health domains, such as computer sicence and user design. The interns suc- cessfully developed three applications that demonstrated a value-add to chemical surveillance-ChemAnalyzer (text analytics), RetroSpect (retrospective analysis of chemical events), and ToxicBusters (geo-based trend analytics). These applications will be the basis for the first chemical surveillance application to be incorporated into the DTRA Biosurveillance Ecosystem (BSVE).

\section{Introduction}

Pacific Northwest National Laboratory (PNNL), on behalf the Defense Threat Reduction Agency (DTRA; project number CB10190), hosts an annual intern- based web app development contest. Previous competitions have focused on mobile biosurveillance applications. The 2016 competition pivoted away from biosurveillance to focus on addressing challenges within the field of chemical surveillance and increasing public health chemical situational awareness. The result of the app will be integrated within the DTRA BSVE.

\section{Methods}

PNNL hosted nine graduate interns for a 10 -week period in the summer of 2016 as participants in a summer web application development contest. Students were drawn from such fields as software engineering and user experience and design and placed into three teams of three students. The challenge presented to the interns was to design and develop a fully-functional web application that would address a critical need within the chemical surveillance community. The interns developed their own ideas (vetted by PNNL and DTRA), discovered and inte- grated their own data sources, and produced their own visualizations and an- alytics, independent of any assistence outside of that provided in an advisory capacity. The competition end with a judging event with a panel of subject matter experts and cash awards were distributed to the teams.

\section{Results}

Each team produced a unique application. Although there was mild overlap between some of the ideas, the applications were developed independently and each reflected the unique contributions of the teams. ChemAnalyzer is a text-analytics platform designed to facilitate more data- driven decision, given a corpus of text data about a chemical event. Their plat- form provided the ability to automatically identify and highlight key words in documents related to chemical events. The keywords are drawn from an on- tology installed with the system, as well as any user-identified keywords. The ChemAnalyzer team finished in third place. The RetroSpect team developed a visual analytic tool for performing retrospec- tive analysis and monitoring of chemical events. Their app provided the ability to search and analyze past events, as well as visualization of state and county information for the recorded chemical events. The RetroSpect team finished in second place. The Toxicbusters team - the winners of the competition - created a geo-based situational awareness tool for tracking chemical events. Their app featured an updateable map overlay, search functionality for finding specific or related events, incident and city/state/national-level statistics and trends, as well as news and social media integration based on keywords related to chemical surveillance.

\section{Conclusions}

Each of the apps developed by the teams provides value to an analyst tasked with monitoring chemical events. The apps integrated unique data sources to provides a full picture of a chemical event, and its effects upon the surrounding population. This integrated analytics provides a valuable benefit over existing workflows, where analysts must monitor news, social, and other information sources manually for real-time information. The apps developed by these interns are designed to enable identification and analysis of the incident as quickly as possible, allowing for more timely assessments of the incident and its impacts. The web app development contest provided a unique opportunity for students to learn about the emerging needs in chemical surveillance as it relates to health sit- uational awareness. Students were drawn from a variety of fields and were tasked with developing novel web apps addressing some of the most pressing challenges in the field of chemical surveillance. The ideas generated by the students will help form the basis for future chemical surveillance application development to be integrated with the DTRA BSVE.

\section{Keywords}

chemical surveillance; public health; data visualization; data science

\section{${ }^{*}$ Michael J. Henry}

E-mail: michael.j.henry@pnnl.gov 